\title{
Approximating Common Fixed Points of Two Sequences of Uniformly Quasi-lipschitzian Mappings in Convex Cone Metric Spaces
}

\author{
Byung-Soo Lee \\ Department of Mathematics, Kyungsung University, Busan 608-736, Korea \\ ${ }^{*}$ Corresponding Author: bslee@ks.ac.kr
}

Copyright (C)2013 Horizon Research Publishing All rights reserved.

\begin{abstract}
A convex cone metric space is a cone metric space with a convex structure. In this paper, we extend an Ishikawa type iterative scheme with errors to approximate a common fixed point of two sequences of uniformly quasi-Lipschitzian mappings to convex cone metric spaces. Our result generalizes Theorem 2 in [1].
\end{abstract}

Keywords Convex Structure, Convex Cone Metric Spaces, Ishikawa Type Iterative Scheme, Common Fixed Point, Uniformly Quasi-lipschitzian Mappings, Asymptoically Quasi-nonexpansive Mappings, Asymptotically Nonexpansive Mappings

2010 Mathematics Subject Classification : 47H09, 47H10

\section{Introduction and Preliminaries}

It is desiralble to add the convex structure in $[3,6]$ to cone metric spaces and consider an Ishikawa type iterative scheme with errors to approximate a common fixed point of two sequences of uniformly quasi-Lipschitzian mappings in convex cone metric spaces.

Throughout this paper, $E$ is a normed vector space with a normal solid cone $P$.

Definition 1.1. [2] A nonempty subset $P$ of $E$ is called a cone if $P$ is closed, $P \neq\{\theta\}$, for $a, b \in \mathbb{R}^{+}=[0, \infty)$ and $x, y \in P, a x+b y \in P$ and $P \cap\{-P\}=\{\theta\}$. We define a partial ordering $\preceq$ in $E$ as $x \preceq y$ if $y-x \in P . x<<y$ indicates that $y-x \in \operatorname{int} P$ and $x \prec y$ means that $x \preceq y$ but $x \neq y$. A cone $P$ is said to be solid if its interior int $P$ is nonempty. A cone $P$ is said to be normal if there exists a positive number $k$ such that for $x, y \in P, 0 \preceq x \preceq y$ implies $\|x\| \leq k\|y\|$. The least positive number $k$ is called the normal constant of $P$.

Definition 1.2. [2] Let $X$ be a nonempty set. A mapping $d: X \times X \rightarrow(E, P)$ is called a cone metric if (i) for $x, y \in X, 0 \preceq d(x, y)$ and $d(x, y)=\theta$ iff $x=y$, (ii) for $x, y \in X, d(x, y)=d(y, x)$ and (iii) for $x, y, z \in X$, $d(x, y) \preceq d(x, z)+d(z, y)$. A nonempty set $X$ with a cone metric $d: X \times X \rightarrow(E, P)$ is called a cone metric space denoted by $(X, d)$, where $P$ is a solid normal cone.

Definition 1.3. [5] Let $(X, d)$ be a cone metric space and $T:(X, d) \rightarrow(X, d)$ a mapping.

(i) $T$ is said to be asymptotically nonexpansive if there exists a sequence $\left\{k_{n}\right\}$ in $[1, \infty)$ such that $\lim _{n \rightarrow \infty} k_{n}=1$ with $d\left(T^{n} x, T^{n} y\right) \preceq k_{n} \cdot d(x, y)$ for all $x, y \in X(n \in \mathbb{N} \cup\{0\})$.

(ii) $T$ is said to be asymptotically quasi-nonexpansive if there exists a sequence $\left\{k_{n}\right\}$ in $[1, \infty)$ such that $\lim _{n \rightarrow \infty} k_{n}=$ 1 with $d\left(T^{n} x, p\right) \preceq k_{n} \cdot d(x, p)$ for all $x \in X$ and $p \in F(T)$, the set of fixed points of $T(n \in \mathbb{N} \cup\{0\})$.

(iii) $T$ is said to be uniformly quasi-Lipschitzian if there exists $L>0$ such that $d\left(T^{n} x, p\right) \preceq L \cdot d(x, p)$ for all $x \in X$ and $p \in F(T)(n \in \mathbb{N} \cup\{0\})$.

Remark 1.1. If $F(T) \neq \emptyset$, then (i) implies (ii), and (ii) implies (iii) by putting $L=\sup _{n \geq 0} k_{n}<\infty$, but not conversely from some examples shown in metric spaces [8].

This research was supported by Kyungsung University Research Grants in 2013. 
Now we consider the convex structure in cone metric spaces.

Definition 1.4. Let $(X, d)$ be a cone metric space. A mapping $W: X^{3} \times I^{3} \rightarrow X$ is called a convex structure on $X$ if $d\left(W\left(x, y, z, a_{n}, b_{n}, c_{n}\right), u\right) \preceq a_{n} \cdot d(x, u)+b_{n} \cdot d(y, u)+c_{n} \cdot d(z, u)$ for real sequences $\left\{a_{n}\right\},\left\{b_{n}\right\}$ and $\left\{c_{n}\right\}$ in $I=[0,1]$ satisfying $a_{n}+b_{n}+c_{n}=1(n \in \mathbb{N})$ and $x, y, z$ and $u \in X$. A cone metric space $(X, d)$ with a convex structure $W$ is called a convex cone metric space and denoted as $(X, d, W)$. A nonempty subset $C$ of a convex cone metric space $(X, d, W)$ is said to be convex if $W(x, y, z, a, b, c) \in C$ for all $x, y, z \in C$ and $a, b, c \in I$.

Definition 1.5. Let $(X, d)$ be a cone metric space, $\left\{x_{n}\right\}$ be a sequence in $X$ and $x \in X$.

(i) If for any $c \in E$ with $0<<c$, there exists a natural number $N$ such that for all $n>N, d\left(x_{n}, x\right)<<c$, then $\left\{x_{n}\right\}$ is said to converge to $x$ and denoted as $\lim _{n \rightarrow \infty} x_{n}=x$ or $x_{n} \rightarrow x($ as $n \rightarrow \infty)$.

(ii) If for any $c \in E$ with $0<<c$, there exists a natural number $N$ such that for all $n, m>N, d\left(x_{n}, x_{m}\right)<<c$, then $\left\{x_{n}\right\}$ is called a Cauchy sequence in $X$.

(iii) $(X, d)$ is said to be complete if every Cauchy sequence converges.

Proposition 1.1. [2] Let $\left\{x_{n}\right\}$ be a sequence in a cone metric space $(X, d)$ and $P$ be a normal cone. Then

(i) $\left\{x_{n}\right\}$ converges to $x$ in $X$ if and only if $d\left(x_{n}, x\right) \rightarrow 0($ as $n \rightarrow \infty)$ in $E$.

(ii) $\left\{x_{n}\right\}$ is a Cauchy sequence if and only if $d\left(x_{n}, x_{m}\right) \rightarrow 0($ as $n, m \rightarrow \infty)$ in $E$.

Let $C$ be a nonempty convex subset of a convex cone metric space $(X, d, W)$. Let $S_{i}, T_{i}: C \rightarrow C$ be uniformly quasi-Lipschitzian mappings with Lipschitzian constants $L_{i}>0$ and $L_{i}^{\prime}>0$, respectively $(i \in \mathbb{N})$.

Let $\left\{u_{n}\right\}$ and $\left\{v_{n}\right\}$ be sequences in $C$ and $\left\{a_{n}\right\},\left\{b_{n}\right\},\left\{c_{n}\right\},\left\{a_{n}^{\prime}\right\},\left\{b_{n}^{\prime}\right\}$ and $\left\{c_{n}^{\prime}\right\}$ be sequences in $[0,1]$ with $a_{n}+b_{n}+c_{n}=a_{n}^{\prime}+b_{n}^{\prime}+c_{n}^{\prime}=1(n \in \mathbb{N} \cup\{0\})$.

For any given $x_{0} \in C$, a sequence $\left\{x_{n}\right\}$ defined as

$$
\left\{\begin{array}{l}
x_{n+1}=W\left(x_{n}, S_{n+1}^{n} y_{n}, u_{n}, a_{n}^{\prime}, b_{n}^{\prime}, c_{n}^{\prime}\right), \\
y_{n}=W\left(x_{n}, T_{n+1}^{n} x_{n}, v_{n}, a_{n}, b_{n}, c_{n}\right),
\end{array}\right.
$$

is called the Ishikawa type iteration process with errors for two sequences $\left\{S_{i}\right\}_{i \in \mathbb{N}}$ and $\left\{T_{i}\right\}_{i \in \mathbb{N}}$.

In this paper, we present more generalized convergence theorem concerning Ishikawa type iterative schemes with errors for approximating a common fixed point of two sequences of uniformly quasi-Lipschitzian mappings in convex cone metric spaces by comparing the convergence theorems considered in convex metric spaces [5, 7, 8]. Our result generalizes Theorem 2 in [1].

\section{Main Results}

Lemma 2.1. [4] Let $\left\{p_{n}\right\},\left\{q_{n}\right\}$ and $\left\{r_{n}\right\}$ be sequences of nonnegative real numbers with $\sum_{n=1}^{\infty} q_{n}<\infty$ and $\sum_{n=1}^{\infty} r_{n}<$ $\infty$. If $p_{n+1} \leq\left(1+q_{n}\right) \cdot p_{n}+r_{n} \quad(n \in \mathbb{N} \cup\{0\})$, then

(i) $\lim _{n \rightarrow \infty} p_{n}$ exists, and

(ii) If $\underset{n \rightarrow \infty}{\lim _{n \rightarrow \infty}} p_{n}=0$, then $\lim _{n \rightarrow \infty} p_{n}=0$.

Theorem 2.2 Let $d: X \times X \rightarrow(E, P)$ be a cone metric, where $P$ is a solid normal cone with the normal constant $k$. Let $C$ be a nonempty closed convex subset of complete convex cone metric space $(X, d, W)$ and $S_{i}, T_{i}: C \rightarrow C$ be uniformly quasi-Lipschitzian mappings with constants $L_{i}>0$ and $L_{i}^{\prime}>0$, respectively $(i \in \mathbb{N})$. Suppose that $F:=\left(\bigcap_{i=1}^{\infty} F\left(S_{i}\right)\right) \cap\left(\bigcap_{i=1}^{\infty} F\left(T_{i}\right)\right)$ is nonempty and bounded. Let $\left\{x_{n}\right\}$ be a sequence in $C$ defined by $(1.1)$ with bounded sequences $\left\{u_{n}\right\}$ and $\left\{v_{n}\right\}$. Let $\left\{a_{n}\right\},\left\{b_{n}\right\},\left\{c_{n}\right\},\left\{a_{n}^{\prime}\right\},\left\{b_{n}^{\prime}\right\}$ and $\left\{c_{n}^{\prime}\right\}$ be real sequences in $I$ with $a_{n}+b_{n}+c_{n}=a_{n}^{\prime}+b_{n}^{\prime}+c_{n}^{\prime}=1 \quad(n \in \mathbb{N} \cup\{0\})$ such that $\sum_{n=0}^{\infty}\left(b_{n}^{\prime}+c_{n}^{\prime}\right)<\infty$.

Assume that $\left\{L_{i}: i \in \mathbb{N}\right\}$ and $\left\{L_{i}^{\prime}: i \in \mathbb{N}\right\}$ are bounded.

Then the followings are equivalent;

(i) $\left\{x_{n}\right\}$ converges to a common fixed point $p \in F$,

(ii) $\varliminf_{n \rightarrow \infty} d\left(x_{n}, F\right)=0$, where $d(x, F)=\inf _{q \in F} d(x, q)$.

Proof. Obviously, (i) implies (ii). Now we consider that (ii) implies (i).

(1) Firstly, we show that $\lim _{n \rightarrow \infty} d\left(x_{n}, F\right)=0$. 
Approximating Common Fixed Points of Two Sequences of Uniformly Quasi-lipschitzian Mappings in Convex

For any $p \in F$, from (1.1) we have

$$
\begin{aligned}
d\left(x_{n+1}, p\right) & =d\left(W\left(x_{n}, S_{n+1}^{n} y_{n}, u_{n}, a_{n}^{\prime}, b_{n}^{\prime}, c_{n}^{\prime}\right), p\right) \\
& \preceq a_{n}^{\prime} \cdot d\left(x_{n}, p\right)+b_{n}^{\prime} \cdot d\left(S_{n+1}^{n} y_{n}, p\right)+c_{n}^{\prime} \cdot d\left(u_{n}, p\right) \\
& \preceq a_{n}^{\prime} \cdot d\left(x_{n}, p\right)+b_{n}^{\prime} \cdot L \cdot d\left(y_{n}, p\right)+c_{n}^{\prime} \cdot d\left(u_{n}, p\right)
\end{aligned}
$$

and

$$
\begin{aligned}
d\left(y_{n}, p\right) & =d\left(W\left(x_{n}, T_{n+1}^{n} x_{n}, v_{n}, a_{n}, b_{n}, c_{n}\right), p\right) \\
& \preceq a_{n} \cdot d\left(x_{n}, p\right)+b_{n} \cdot d\left(T_{n+1}^{n} x_{n}, p\right)+c_{n} \cdot d\left(v_{n}, p\right) \\
& \preceq a_{n} \cdot d\left(x_{n}, p\right)+b_{n} \cdot L \cdot d\left(x_{n}, p\right)+c_{n} \cdot d\left(v_{n}, p\right) \\
& =\left(a_{n}+b_{n} \cdot L\right) \cdot d\left(x_{n}, p\right)+c_{n} \cdot d\left(v_{n}, p\right),
\end{aligned}
$$

where $L=\max \left\{\sup _{i \in \mathbb{N}} L_{i}, \sup _{i \in \mathbb{N}} L_{i}^{\prime}\right\}$.

So,

$$
\begin{aligned}
d\left(x_{n+1}, p\right) & \preceq a_{n}^{\prime} \cdot d\left(x_{n}, p\right)+b_{n}^{\prime} \cdot L \cdot\left[\left(a_{n}+b_{n} \cdot L\right) \cdot d\left(x_{n}, p\right)+c_{n} \cdot d\left(v_{n}, p\right)\right]+c_{n}^{\prime} \cdot d\left(u_{n}, p\right) \\
& \preceq\left[1+b_{n}^{\prime} \cdot L \cdot(1+L)\right] \cdot d\left(x_{n}, p\right)+c_{n}^{\prime} \cdot d\left(u_{n}, p\right)+b_{n}^{\prime} \cdot c_{n} \cdot L \cdot d\left(v_{n}, p\right) \\
& \preceq\left[1+b_{n}^{\prime} \cdot L \cdot(1+L)\right] \cdot d\left(x_{n}, p\right)+\left[d\left(u_{n}, p\right)+L \cdot d\left(v_{n}, p\right)\right]\left(b_{n}^{\prime}+c_{n}^{\prime}\right) \\
& =\left[1+b_{n}^{\prime} \cdot L \cdot(1+L)\right] \cdot d\left(x_{n}, p\right)+\eta_{n} \cdot u,
\end{aligned}
$$

where $u=\sup _{p \in F, n \geq 1}\left\{d\left(u_{n}, p\right)+L \cdot d\left(v_{n}, p\right)\right\}$ and $\eta_{n}=b_{n}^{\prime}+c_{n}^{\prime}(n \in \mathbb{N} \cup\{0\})$.

Thus, by the normality of $P$, for the normal constant $k>0$

$$
\left\|d\left(x_{n+1}, p\right)\right\| \leq k \cdot\left[1+b_{n}^{\prime} \cdot L \cdot(1+L)\right] \cdot\left\|d\left(x_{n}, p\right)\right\|+k \cdot\|u\| \cdot \eta_{n} .
$$

Since $p$ is an arbitrary point in $F$,

$$
\left\|d\left(x_{n+1}, F\right)\right\| \leq k \cdot\left[1+b_{n}^{\prime} \cdot L \cdot(1+L)\right]\left\|\cdot d\left(x_{n}, F\right)\right\|+k \cdot\|u\| \cdot \eta_{n}
$$

Since $\sum_{n=0}^{\infty} \eta_{n}=\sum_{n=0}^{\infty}\left(b_{n}^{\prime}+c_{n}^{\prime}\right)$ is finite, by Lemma 2.1, $\lim _{n \rightarrow \infty}\left\|d\left(x_{n}, F\right)\right\|$ exists, so $\lim _{n \rightarrow \infty} d\left(x_{n}, F\right)$ exists. Now $\varliminf_{n \rightarrow \infty} d\left(x_{n}, F\right)=0$ implies $\lim _{n \rightarrow \infty} d\left(x_{n}, F\right)=0$.

(2) Secondly, we show that $\left\{x_{n}\right\}$ is a Cauchy sequence in $C$.

Since $\lim _{n \rightarrow \infty}\left\|d\left(x_{n}, F\right)\right\|=0$ and $\sum_{n=0}^{\infty} \eta_{n}<\infty$, for any positive real number $\varepsilon$, there exists a natural number $N_{0} \in \mathbb{N}$ such that $\left\|d\left(x_{n}, F\right)\right\| \leq \frac{\varepsilon}{4 M_{1}}$ for $n \geq N_{0}$ and $\sum_{n=N_{0}}^{\infty} \eta_{n} \leq \frac{\varepsilon}{4\|u\| \cdot M_{1}}$, where $M_{1}=e^{L \cdot(1+L) \sum_{k=0}^{\infty} b_{k}^{\prime}}$.

In particular, there exist a point $p_{1} \in F$ and a positive integer $N_{1}>N_{0}$ such that

$$
\left\|d\left(x_{N_{1}}, p_{1}\right)\right\| \leq \frac{\varepsilon}{4 M_{1}}
$$

On the other hand, by the fact that

$$
\left\|d\left(x_{n+1}, p\right)\right\| \leq k \cdot\left(1+b_{n}^{\prime} \cdot L \cdot(1+L)\right)\left\|\cdot d\left(x_{n}, p\right)\right\|+k \cdot\|u\| \cdot \eta_{n}
$$


and the inequality $1+x \leq e^{x}$ for $x \geq 0$, we have

$$
\begin{aligned}
& \left\|d\left(x_{n+m}, p\right)\right\| \leq k_{1} \cdot\left(1+b_{n+m-1}^{\prime} \cdot L \cdot(1+L)\right) \cdot\left\|d\left(x_{n+m-1}, p\right)\right\|+k_{1} \cdot\|u\| \cdot \eta_{n+m-1} \\
& \leq k_{1} \cdot e^{b_{n+m-1}^{\prime} \cdot L \cdot(1+L)} \cdot\left\|d\left(x_{n+m-1}, p\right)\right\|+k_{1} \cdot\|u\| \cdot \eta_{n+m-1} \\
& \leq k_{1} \cdot e^{b_{n+m-1}^{\prime} \cdot L \cdot(1+L)} \cdot\left[k_{2} \cdot\left(1+b_{n+m-2}^{\prime} \cdot L \cdot(1+L)\right) \cdot\left\|d\left(x_{n+m-2}, p\right)\right\|\right. \\
& \left.+k_{2} \cdot\|u\| \cdot \eta_{n+m-2}\right]+k_{1} \cdot\|u\| \cdot \eta_{n+m-1} \\
& \leq k_{1} \cdot e^{b_{n+m-1}^{\prime} \cdot L(1+L)}\left[k_{2} \cdot e^{b_{n+m-2}^{\prime} \cdot L(1+L)} \cdot\left\|d\left(x_{n+m-2}, p\right)\right\|+k_{2} \cdot\|u\| \cdot \eta_{n+m-2}\right] \\
& +k_{1} \cdot\|u\| \cdot \eta_{n+m-1} \\
& \leq k_{1} \cdot k_{2} \cdot e^{\left(b_{n+m-1}^{\prime}+b_{n+m-2}^{\prime}\right) L \cdot(1+L)} \cdot\left[k_{3} \cdot e^{b_{n+m-3}^{\prime} \cdot L \cdot(1+L)}\left\|d\left(x_{n+m-3}, p\right)\right\|\right. \\
& \left.+k_{3} \cdot\|u\| \cdot \eta_{n+m-3}\right]+k_{1} \cdot k_{2} \cdot e^{b_{n+m-1}^{\prime} \cdot L \cdot(1+L)} \cdot\|u\| \cdot \eta_{n+m-2}+k_{1} \cdot\|u\| \cdot \eta_{n+m-1} \\
& =k_{1} \cdot k_{2} \cdot k_{3} \cdot e^{\left(b_{n+m-1}^{\prime}+b_{n+m-2}^{\prime}+b_{n+m-3}^{\prime}\right) \cdot L \cdot(1+L)}\left\|d\left(x_{n+m-3}, p\right)\right\| \\
& +k_{1} \cdot k_{2} \cdot k_{3} \cdot e^{\left(b_{n+m-1}^{\prime}+b_{n+m-2}^{\prime}\right) L \cdot(1+L)} \cdot\|u\| \cdot \eta_{n+m-3} \\
& +k_{1} \cdot k_{2} \cdot e^{b_{n+m-1}^{\prime} \cdot L \cdot(1+L)} \cdot\|u\| \cdot \eta_{n+m-2}+k_{1} \cdot\|u\| \cdot \eta_{n+m-1} \\
& \leq \prod_{j=1}^{4} k_{j} \cdot e^{L \cdot(1+L) \cdot \sum_{j=1}^{4} b_{n+m-j}^{\prime}}\left\|d\left(x_{n+m-4}, p\right)\right\| \\
& +\prod_{j=1}^{4} k_{j} \cdot e^{L \cdot(1+L) \sum_{j=1}^{3} b_{n+m-j}^{\prime}} \cdot\|u\| \cdot \eta_{n+m-4} \\
& +\prod_{j=1}^{3} k_{j} \cdot e^{L \cdot(1+L) \sum_{j=1}^{2} b_{n+m-j}} \cdot\|u\| \cdot \eta_{n+m-3} \\
& +k_{1} \cdot k_{2} \cdot e^{L(1+L) b_{n+m-1}^{\prime}} \cdot\|u\| \cdot \eta_{n+m-2} \\
& +k_{1} \cdot\|u\| \cdot \eta_{n+m-1} \\
& \vdots \\
& \leq \prod_{j=1}^{m} k_{j} \cdot e^{L \cdot(1+L) \sum_{j=1}^{m} b_{n+m-j}^{\prime}} \cdot\left\|d\left(x_{n}, p\right)\right\|+\prod_{j=1}^{m} k_{j} \cdot e^{L \cdot(1+L) \sum_{j=1}^{m-1} b_{n+m-j}^{\prime}} \cdot\|u\| \cdot \eta_{n} \\
& +\prod_{j=1}^{m-1} k_{j} \cdot e^{L \cdot(1+L)} \sum_{j=1}^{m-2} b_{n+m-j}^{\prime} \cdot\|u\| \cdot \eta_{n+1} \\
& +\prod_{j=1}^{3} k_{j} \cdot e^{L \cdot(1+L)\left(b_{n+m-1}^{\prime}+b_{n+m-2}^{\prime}\right)} \cdot\|u\| \cdot \eta_{n+m-3} \\
& +k_{1} \cdot k_{2} \cdot e^{L \cdot(1+L) b_{n+m-1}^{\prime}} \cdot\|u\| \cdot \eta_{n+m-2} \\
& +k_{1} \cdot\|u\| \cdot \eta_{n+m-1} \\
& \text { for each corresponding normal constant } k_{i}(i=1 \cdots m) \text {. }
\end{aligned}
$$

Hence $\left\|d\left(x_{n+m}, p\right)\right\| \leq K \cdot M_{1} \cdot\left\|d\left(x_{n}, p\right)\right\|+K \cdot M_{1} \cdot\|u\| \cdot \sum_{j=n}^{n+m-1} \eta_{j}$ where $K=\max \left(\prod_{j=1}^{m} k_{j}, \prod_{j=1}^{m-1} k_{j}, \prod_{j=1}^{m-2} k_{j}, \cdots, k_{1} \cdot k_{2}, k_{1}, 1\right)$. 
Approximating Common Fixed Points of Two Sequences of Uniformly Quasi-lipschitzian Mappings in Convex

Thus we have for $m+n, n>N_{1}$,

$$
\begin{aligned}
\left\|d\left(x_{n+m}, x_{n}\right)\right\| \leq & \left\|d\left(x_{n+m}, p_{1}\right)\right\|+\left\|d\left(x_{n}, p_{1}\right)\right\| \\
\leq & D_{1} \cdot M_{1} \cdot\left\|d\left(x_{N_{1}}, p_{1}\right)\right\|+D_{1} \cdot M_{1} \cdot\|u\| \cdot \sum_{k=N_{1}}^{n+m-1} \eta_{k} \\
& +D_{2} \cdot M_{1} \cdot\left\|d\left(x_{N_{1}}, p_{1}\right)\right\|+D_{2} \cdot M_{1} \cdot\|u\| \cdot \sum_{k=N_{1}}^{n-1} \eta_{k} \\
\leq & \left(D_{1}+D_{2}\right) \cdot M_{1} \cdot \frac{\varepsilon}{4 M_{1}}+\left(D_{1}+D_{2}\right) \cdot M_{1} \cdot\|u\| \cdot \frac{\varepsilon}{4 \cdot M_{1} \cdot\|u\|} \\
= & D \cdot \varepsilon \text { for } D_{1} \text { and } D_{2} \text { obtained as the same method in which } \\
& K \text { is obtained, where D }=\max \left(D_{1}, D_{2}\right) .
\end{aligned}
$$

On the other hand, for $c \in E$ with $0<<c$, there exists a positive number $\delta$ such that for $d \in E$ with $\|d\|<\delta$, $c-d \in$ intP. From the fact that $d\left(x_{n}, x_{m}\right) \rightarrow 0($ as $n, m \rightarrow \infty)$ in $E$, for such $\delta$, there exists a natural number $N$ such that for all $n, m \geq N\left\|d\left(x_{n}, x_{m}\right)\right\|<\delta$. Thus $c-d\left(x_{n}, x_{m}\right) \in$ intP, which means that $\left\{x_{n}\right\}$ is a Cauchy sequence in $C$ by Propostion 1.1. Since $X$ is complete and $C$ is closed, $\left\{x_{n}\right\}$ converges to some point $p^{*}$ in $C$.

(3) Lastly, we show that $p^{*} \in F$. Let $\left\{p_{n}\right\}$ be a sequence in F such that $p_{n} \rightarrow p^{\prime}$.

Since

$$
\begin{aligned}
d\left(p^{\prime}, T_{i} p^{\prime}\right) & \preceq d\left(p^{\prime}, p_{n}\right)+d\left(p_{n}, T_{i} p^{\prime}\right) \\
& =d\left(p^{\prime}, p_{n}\right)+d\left(T_{i} p_{n}, T_{i} p^{\prime}\right) \\
& \preceq d\left(p^{\prime}, p_{n}\right)+L \cdot d\left(p_{n}, p^{\prime}\right) \\
& \rightarrow 0(\text { as } n \rightarrow \infty),
\end{aligned}
$$

$d\left(p^{\prime}, T_{i} p^{\prime}\right)=0$ for $i \in \mathbb{N}$. Similarly, $d\left(p^{\prime}, S_{i} p^{\prime}\right)=0$ for $i \in \mathbb{N}$.

Thus $p^{\prime} \in F$, which means that $F$ is closed. Since $d\left(p^{*}, F\right)=\lim _{n \rightarrow \infty} d\left(x_{n}, F\right)=0$ we have, $p^{*} \in F$, which completes the proof.

Remark 2.1. Taking bounded constants $L_{i}=\sup _{n \geq 0} k_{i_{n}}$ and $L_{i}^{\prime}=\sup _{n \geq 0} k_{i_{n}}^{\prime}$ for asymptotically quasi-nonexpansive mappings and asymptotically nonexpansive mappings such that $\lim _{n \rightarrow \infty} k_{i_{n}}=\lim _{n \rightarrow \infty} k_{i_{n}}^{\prime}(i \in \mathbb{N})=1$, we obtain the same results in Theorem 2.2

Remark 2.2. If $(E, P)$ is a Banach space, then the convex cone metric spaces need not be complete in Theorem 2.2 , we obtain the same results as follows; continued from the fact that $\left\|d\left(x_{n+m}, x_{n}\right)\right\| \leq K \cdot \varepsilon$ in the second part (2) of the proof .

Since $d\left(x_{n}, x_{m}\right) \rightarrow 0($ as $n, m \rightarrow \infty)$ in a Banach space $E$, there exists a point $x$ in $E$ such that $d\left(x_{n}, x\right) \rightarrow 0$ $($ as $n \rightarrow \infty)$.

On the other hand, for $c \in E$ with $0<<c$, there exists a positive number $\delta$ such that for $\|d\|<\delta, c-d \in$ intP. From the fact that $d\left(x_{n}, x\right) \rightarrow 0$ (as $\left.n \rightarrow \infty\right)$ in $E$, for such $\delta$, there exists a natural number $N$ such that $c-d\left(x_{n}, x\right) \in \operatorname{int} P$, which implies that $d\left(x_{n}, x\right)<<c$. Since $C$ is closed, $\left\{x_{n}\right\}$ converges to $x$ in $C$.

Remark 2.3. Restricting a normed linear space $(E, P)$ to a real number system $(\mathbb{R},[0, \infty])$, we obtain the same results as Theorem 2.2 in complete convex metric spaces.

Remark 2.4. By reducing the convex cone metric space $(X, d, W)$ in Theorem 2.2 to the convex metric space in Theorem 2 in [1], but without changing all assumptions in Theorem 2.2, we obtain Theorem 2 in [1] as a direct corollary for the sequnce $\left\{x_{n}\right\}$ defined as

$$
\left\{\begin{array}{l}
x_{n+1}=W\left(x_{n}, T_{n}^{n} y_{n}, u_{n} ; \alpha_{n}, \beta_{n}, \gamma_{n}\right), \\
y_{n}=W\left(x_{n}, S_{n}^{n} y_{n}, v_{n} ; a_{n}, b_{n}, c_{n}\right),
\end{array}\right.
$$

which is the Ishikawa-type iterative sequence with errors considered in [5].

\section{Acknowledgements}

The author is grateful to the reviewer for his(or her) valuable and kind comments improving the manuscript.

\section{REFERENCES}

[1] S. Elmas and M. Ozdemir, Convergence of a general iterative scheme for three infinite families of uniformly quasiLipschitzian mappings in convex metric spaces, Advances in Fixed Point Theory 3(2013) 406-417. 
[2] L.-G. Huang and X. Zhang, Cone metric spaces and fixed point theorems of contractive mappings, J. Math. Anal. Appl. 332(2007) 1468-1476.

[3] B. S. Lee, Strong convergence theorems with a Noor-type iterative scheme in convex metric spaces, Com. Math. Appl. 61(2011) 3218-3225.

[4] Q. H. Liu, Iterative sequences for asymptotically quasi-nonexpansive mappings with errors number, J. Math. Anal. Appl. 259(2001) 18-24.

[5] Q. Y. Liu, Z, B. Liu and N. J. Huang, Approximating the common fixed points of two sequences of uniformly quasiLipschitzian mappings in convex metric spaces, Appl. Math. Comput. 216(2010) 883-889.

[6] W. Takahashi, A convexity in metric space and nonexpansive mappings, Kodai. Math. Rep. 22(1970) 142-149.

[7] Y.X. Tian, Convergence of an Ishikawa type iterative scheme for asymptotically quasi-nonexpansive mappings, Comput. Math. Appl. 49(2005) 1905-1912.

[8] C. Wang and L. W. Liu, Convergence theorems for fixed points of uniformly quasi-Lipschitzian mappings in convex metric spaces, Nonlinear Analysis TMA 70(2009) 2067-2071. 